\title{
Article
}

\section{Prevention Effect of TGF- $\beta$ Type I Receptor Kinase Inhibitor in Esophageal Stricture Formation after Corrosive Burn}

\author{
Min Tae Kim 1,2 and Kun Yung Kim 1,3
}

${ }^{1}$ Biomedical Engineering Research Center, Asan Medical Center, University of Ulsan College of Medicine, 88, Olymic-ro 43-gil, Songpa-gu, Seoul, 138-736 and South Korea

2 Department of Radiologic Technology, Cheju Halla University, Jeju-si, Jeju-do 63092, South Korea.

${ }^{3}$ Department of Radiology and Research Institute of Clinical Medicine of Chonbuk National University-

Biomedical Research Institute of Chonbuk National University Hospital, Jeonju, Korea

Correspondence: Kun Yung Kim. MD, Ph.D. TEL: 82-10-5063-0046; E-mail: kky2kkw@gmail.com

\begin{abstract}
Corrosive burns lead to progressive esophageal stricture and dysphagia. There are many trials to prevent Esophageal stricture formation after corrosive burn. This study aimed to access the effects of EW-7197 on prevention for esophageal stricture formation after corrosive esophageal burn. animal study were classified divided into three groups: a healthy group, a control group (corrosive burn without EW-7197), and a treatment group (corrosive burn with EW-7197). Corrosive esophageal burns were produced using $30 \% \mathrm{NaOH}$ on the lower esophagus. For 3 weeks, the control group received vehicle and the treatment group received $20 \mathrm{mg} / \mathrm{kg} /$ day EW-7197. Treatment efficacy was assessed by measuring the stenosis ratio by esophagogram with contrast media on day 21 . histologic staining was performed to evaluate the fibrosis area ratio, and western blotting was performed to evaluate fibrotic markers. Among 20 rats that underwent surgery, 14 survived. Three in the treatment group died because of esophageal perforation, and three in the control group died due to their debilitating status. The esophageal stenosis ratio was significantly lower in the treatment group than in the control group $(12.1 \pm 9.5 \%$ and $42.2 \pm 8.3 \%$, respectively; $\mathrm{p}=0.001)$. The histologic fibrosis area ratio was also significantly lower in the treatment group $(12.5 \pm 3.0 \%$ and $21.6 \pm 2.1 \%$, respectively; $\mathrm{p}=0.001)$. The treatment group showed lower expressions of profibrogenic proteins such as TGF$\beta 1$, pSmad3, and $\alpha$-SMA. EW-7197 may be a good alternative for the prevention esophageal stricture formation after corrosive burn.
\end{abstract}

Keywords: Corrosive burn; esophageal stricture; TGF- $\beta 1$,

\section{Introduction}

Ingestion of corrosive substances accidentally or in a suicide remains an important cause of esophageal strictures[1, 2]. Esophageal strictures Formation after corrosive burns is a complication of deep esophageal injury, which stimulates excess fibrogenic actions. This leads to progressive esophageal stricture and dysphagia, which significantly decreases the quality of life. A number of therapeutic options had been investigated to prevent the formation of strictures in experimental corrosive esophageal injury models [3-9]. The majority of these therapeutic agents have been used to exert antifibrogenic actions, but the benefits and standard protocols are still controversial [4]. In addition, most of these options have not obtained for clinical use because of suspected toxicities, such as immunosuppression.

The transforming growth factor (TGF) $\beta$ type I receptor kinase inhibitor family has been tested in various animal models about fibrosis [10-14]. EW-7197 (N-[[4-([1,2,4] Triazolo[1,5-a]pyridin-6-yl)-5-(6-methylpyridin-2-yl)-1H-imidazol-2-yl]methyl]-2-fluoroaniline) is a novel orally available TGF- $\beta$ type I receptor kinase inhibitor with high selectivity and low toxicity. EW-7197 has been investigated in several animal models of fibrosis to have antifibrotic and antiproliferative effect $[15,16]$. 
We hypothesized that EW-7197 can suppress stricture formation after corrosive esophageal burn by inhibiting TGF- $\beta$ type I induced deposition of the extracellular matrix (ECM). Therefore, this study aimed to access the effects of EW-7197 on prevention for esophageal stricture formation after corrosive burn.

\section{Materials and Methods}

\subsection{Antifibrotic drug}

EW-7197 phosphate was provided from the Laboratory of Medicinal Chemistry, College of Pharmacy, Ewha Woman's University (Seoul, Korea). The dosage of EW-7197 phosphate was decided based on previous studies $[17,18]$.

\subsection{Animals}

All animal experiments were approved by the Institutional Animal Care and Use Committee at the Asan Medical Center, University of Ulsan College of Medicine (Approval No. 2017-13-246). Thirty Sprague-Dawley rats (9 weeks old, 300-350 g) were divided using a random allocation program (Microsoft, Seattle, WA, USA) into three groups: a healthy group, a corrosive burn without EW-7197 treatment group (the control group), and a corrosive burn with EW-7197 treatment group (the treatment group).

\subsection{Surgical procedure}

Surgical procedure was done as previously described [5]. After $12 \mathrm{~h}$ of fasting, rats were anesthetized by intramuscular injection of $50 \mathrm{mg} / \mathrm{kg}$ zolazepam and tiletamine (Zoletil 50; Virbac, Carros, France) and $10 \mathrm{mg} / \mathrm{kg}$ xylazine (Rompun; Bayer HealthCare, Leverkusen, Germany). A median laparotomy was performed, and a $1.5-\mathrm{cm}$ segment of the lower esophagus was prepared after dissection. A 5 French feeding catheter was placed in the upper part of the lower esophagus through the mouth. The gastroesophageal junction was tied externally with $2 / 0$ silk ligature to prevent to prevent leakage of caustic agent into the stomach. Proximally, just under the diaphragm, the esophagus was tied externally with $2 / 0$ silk ligature to prevent regurgitation and aspiration. A thinner catheter was inserted through the feeding catheter for irrigation. Then, $1 \mathrm{~mL}$ of $30 \% \mathrm{NaOH}$ solution was injected through the thinner catheter for $90 \mathrm{~s}$. Subsequently, the solution was aspirated back, and distilled water was used to irrigate the burned area for $60 \mathrm{~s}$. Intraluminal pressure were carefully controlled during infusion and perfusion until slight translucency of the esophageal wall and branching of vessels were observed. The proximal silk ligature was cut, and the catheter was pulled out under negative pressure. The distal suture was then cut and the laparotomy was finished. All animals were kept on a standard rodent pellet diet with tap water ad libitum after surgery. The treatment group $(\mathrm{n}=10)$ received $20 \mathrm{mg} / \mathrm{kg}$ EW-7197 phosphate by gavage once daily for 3 weeks after surgery. The control group $(n=10)$ received a solution consisting of $0.3 \mathrm{~mL}$ of artificial gastric juice once daily for 3 weeks after surgery. The healthy group $(n=10)$ was not subjected to the surgical procedure and did not receive any treatment. After surgical procedure, behavioral and weight changes were monitored on a weekly. At 3 weeks after surgical procedure, all rats sacrificed for histopathology and western blotting by administering inhalable pure carbon dioxide.

\subsection{Fluoroscopic esophagogram}

Fluoroscopic esophagogram with contrast media was performed at 3 weeks after the procedure in all groups. Esophagogram was performed under the same anesthesia method as described above, using a contrast medium (Ultravist 300; Schering Korea, Anseong, Korea). The stenosis ratio was evaluated by measuring the axial diameter in both the upper non-damaged area and the lower stenosis area. Quantitative determination was performed using Image J software.

\subsection{Esophageal histopathology}


The stenosed portion of the lower esophagus was obtained 3 weeks after corrosive burn. Tissue samples were fixed in 10\% neutral-buffered formalin for $24 \mathrm{~h}$, which was then embedded in paraffin, sectioned into 5- $\mu \mathrm{m}$ sections, and stained with Masson's trichrome. The healthy rats underwent the same procedure. The total areas encircling the outer margins of the esophagus, luminal area, and submucosal collagen area of the axial section were measured to determine the fibrosis area ratio. This ratio was defined by the formula (submucosal collagen area) / (total area - luminal area). All histopathologic images were analyzed with the Image J software.

\subsection{Western blotting}

Western blotting was performed using 10\% sodium dodecyl sulfate polyacrylamide gel electrophoresis. Primary antibodies were against Smad3 (1:1000; Cell Signaling Technology [CST], Danvers, MA, USA), pSmad3 (1:1000; CST), $\alpha$-smooth muscle actin ( $\alpha$-SMA; 1:300; Abcam, Cambridge, Cambridgeshire, UK), TGF- $\beta 1$ (1:1000; CST), and $\beta$-actin $(1: 1000 ; C S T)$. After incubation with horseradish peroxidase-conjugated secondary antibodies (1:1000; Jackson ImmunoResearch, West Grove, PA, USA) and enhanced chemiluminescence western blotting detection reagents (Amersham Biosciences, Little Chalfont, Buckinghamshire, UK), immunoreactive bands were visualized using an Ez-Capture MG (ATTO Corporation, Tokyo, Japan). Densitometric values of the bands were quantified and expressed as their ratio to $\beta$-actin using CS Analyzer software (ATTO Corporation). The healthy group was used to ascertain normal values.

\subsection{Statistical analysis}

Differences between the groups were analyzed using the Kruskal-Wallis test and the Mann-Whitney U test. Body weight measurement was done in the rats that survived the full experimental period. A p value of $<0.05$ was considered statistically significant. Statistical analyses were performed using the SPSS software (version 23.0; SPSS, IBM, Chicago, IL, USA).

\section{Results}

Each procedure taken approximately 30 min for completion. Among 20 rats that underwent surgery, 14 survived the complete experimental period of 3 weeks. A total of six rats died: three in the treatment group, from esophageal perforation, and three in the control group, from a debilitating status related to dysphagia, in the second week. The 21-day survival rates were $70 \%$ in both the control and treatment groups. Baseline body weight was no statistically significantly difference between the control and treatment groups $(343.2 \pm 32.8$ and $329.5 \pm 22.2$, respectively; $p=0.490)$, and body weight gain was no significantly significantly difference between the two groups $(12.6 \pm 72.8$ and $-19.7 \pm 50.8$, respectively; $\mathrm{p}=0.384)$. The prevalence rate of esophageal stricture-related symptoms (which included hypersalivation, hemoptysis, and stridor) was 50\% ( $\mathrm{n}=7$; three rats in the control group and four in the treatment group). The remaining seven rats were free from esophageal stricture-related symptoms.

\subsection{Effect of the drug on esophageal stenosis}

Figure 1 shows the esophagographic stenosis ratio, as determined by fluoroscopic esophagogram. The stenosis ratios of the esophagus in the healthy group, control group, and treatment group were $1.3 \pm 5.2 \%, 42.2 \pm 8.3 \%$, and $12.1 \pm 9.5 \%$, respectively. The treatment group had a significantly lower stenosis ratio than the control group $(p=0.001)$. 


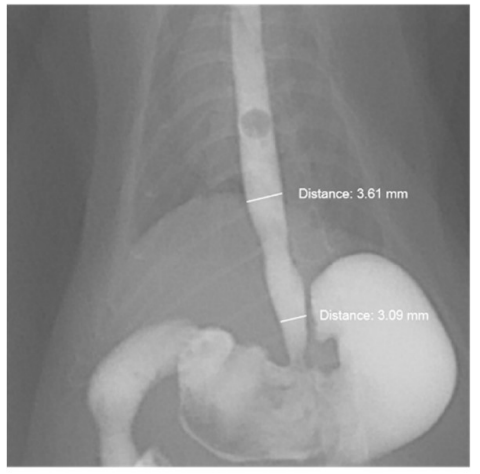

Fig 1-A

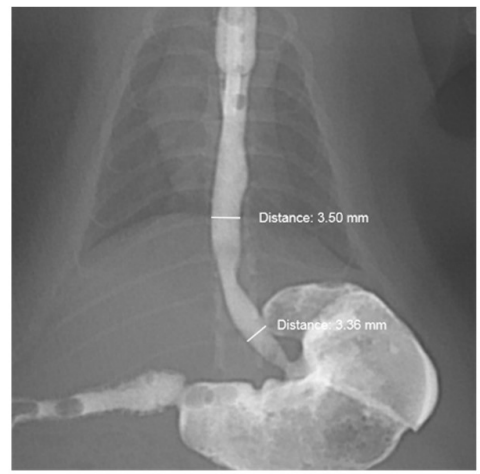

Fig 1-B

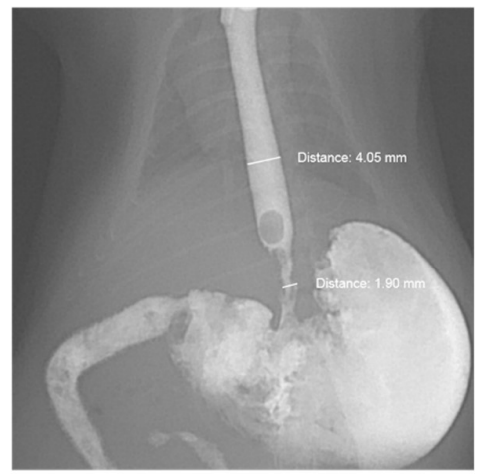

Fig 1-C

Figure 1. Fluoroscopic esophagogram with contrast media at 3 weeks after surgery. The stenosis ratio was determined by comparing the axial diameter in both the upper undamaged area and the lower stenosis area. Healthy rat; rat with corrosive burn but without TGF- $\beta 1$ inhibitor treatment (control); rat with corrosive burn and with TGF- $\beta 1$ inhibitor treatment (treatment) (A-C, respectively). The figure demonstrates significantly less esophageal stenosis in the treatment group compared to the control group $(\mathrm{p}=0.001)$.

\subsection{Effect of the drug on fibrosis}

Figure 2 shows the fibrosis area ratio, as determined by histopathologic results. The fibrosis area ratios of the esophagus in the healthy group, control group, and treatment group were $10.6 \pm 3.2 \%, 21.6 \pm 2.1 \%$, and $12.5 \pm 3.0 \%$, respectively. The treatment group had a significantly lower fibrosis area ratio than the control group $(p=0.001)$.

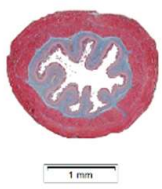

Fig 2-A

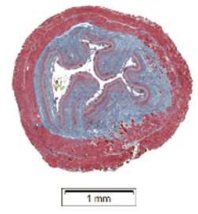

Fig 2-B

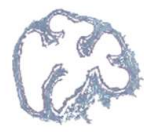

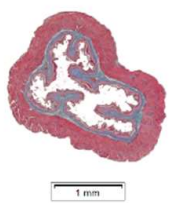

Fig 2-C

Figure 2. Masson's trichrome stain was used for fibrosis determination. Healthy rat; rat with corrosive burn but without TGF- $\beta 1$ inhibitor treatment (control); rat with corrosive burn and with TGF- $\beta 1$ inhibitor treatment (treatment) (A-C, respectively). The fibrosis area ratio was significantly lower in the treatment group than that in the control group ( $p=0.001)$.

\subsection{Effect of the drug on protein expression}

Figure 3 shows the western blotting results. EW-7197 treatment for 3 weeks significantly suppressed the expression of the profibrogenic proteins TGF- $\beta 1$, pSmad3, and $\alpha$ SMA. We found that the expression of these proteins increased significantly in the control group compared to the treatment group $(\mathrm{p}<0.05)$. 


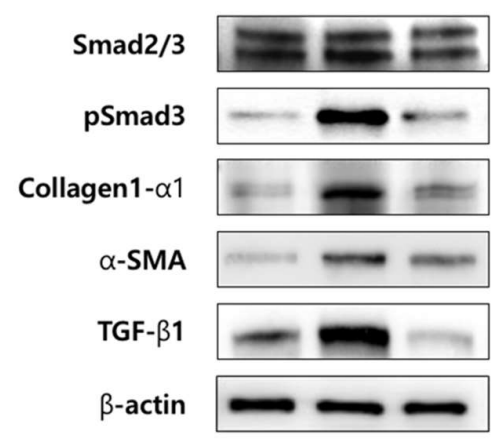

Fig 3-A

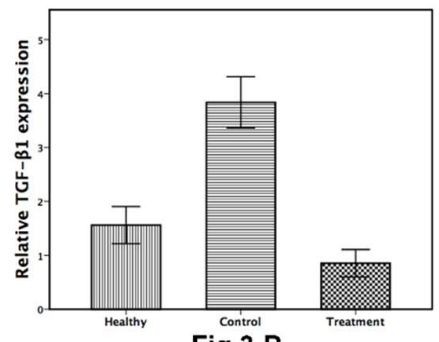

Fig 3-B

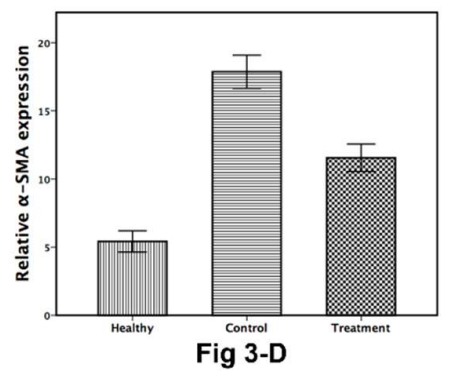

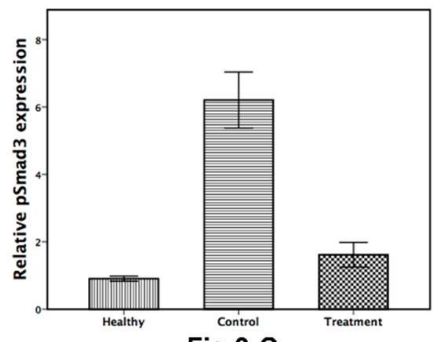

Fig 3-C

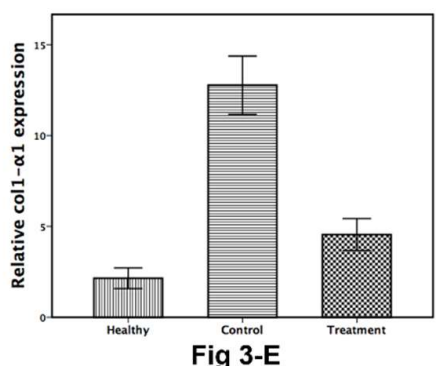

Figure 3. Representative western blotting results (A). Quantitative expression ratio of TGF- $\beta 1$, pSmad3, and $\alpha$-SMA were significantly decreased in the treatment group compared to the control group (B-E, respectively) $(p=0.001)$.

\section{Discussion}

Stricture formation in the chronic stage of corrosive esophagitis is a commonly encountered complication. It requires long and repetitive treatment. Some investigators advocate insertion of a silicone stent or a nasogastric tube for the acute and subacute stages after a corrosive burn to reduce stricture formation $[19,20]$. However, Gumaste VV et al. do not advocate these practice because stent-induced mechanical injury may contribute to substantial complications [21]. Although steroids are clinically used, it exposes patients to many side effects and controversial results. There is no established medical treatment during the acute stage that can prevent further stricture formation. Experimentally, many treatment options have been used to reduce stricture formation [3-9, 22]; however, only a few of them are used clinically.

Antifibrotic agents have been the main focus of preventing esophageal stricture. Recently, Orozco-Perez et al. reported that pirfenidone, an ECM degradation modulator, prevented esophageal stricture formation after corrosive burn in rats [9]. The collagen deposition in the tissue is controlled between the synthesis and decomposition of various types of collagen components of the ECM[23]. Collagen synthesis is enhanced by TGF- $\beta 1$. It plays an important role in the transition from inflammatory phase to proliferative phase, particularly in fibroblasts and collagen deposition, indicating the pathophysiological pathway of stenosis formation[22].

Kim et al. reported that blocking the biological action of TGF- $\beta$ may be alternative treatment option to inhibit significant stricture formation [14,24,25]. More recently, Jun et al. reported that EW-7197 suppressed fibrotic tissue hyperplasia formation with esophageal stent in a rat esophageal model [23]. They suggested it has potential treatment option as an antifibrotic drug via its ability to inhibit TGF- $\beta$ signaling [23]. EW-7197 is a selective oral bioavailable inhibitor of TGF- $\beta 1$, which has been studied as an antifibrotic agent $[15$, $16,18]$. In this context, we hypothesized that a TGF- $\beta 1$ inhibitor could suppress stricture formation after corrosive esophageal burn by inhibiting TGF- $\beta 1 / \mathrm{Smad} 3$-induced deposition of the ECM.

Our study demonstrated that the stenosis ratio determined by the fluoroscopic study and the fibrosis area ratio determined by histology analysis were prominent lower in the treatment group than those in the control group. Although we did not perform quantitative analysis, the expression patterns of profibrogenic proteins such as pSmad3 and $\alpha$ SMA were suppressed in the treatment group in our western blot analysis. This result 
indicate that EW-7197 suppressed stricture formation by inhibiting TGF- $\beta 1 /$ Smad3-induced ECM synthesis. Our results consent with previous studies performed in various animal models [14-16, 18].

However, our results were not directly connected to the improvement of survival or symptoms. Survival rates at 3 weeks after surgery were the same (70\%) in both groups. In addition, the occurrence rate of esophageal stricture-related symptoms was $42.9 \%$ in the control group and $57.1 \%$ in the treatment group. Moreover, three rats in the treatment group died in the first week after surgery due to esophageal perforation. TGF- $\beta$ could be responsible not only for wound scarring but also for wound healing. The key roles of TGF$\beta$ in wound healing including production and remodeling of the ECM, which is crucial for tissue damage repair. We surmise that the treatment group experienced with delayed wound healing and fibrosis inhibition in the injured segment, which led to esophageal perforation. It is already well known that fibroblast proliferation and collagen deposition occurs in the second week after injury, representing the biological pathway of stricture formation [25]. More research is needed to investigate complex etiological cause in acute and subacute periods of corrosive burns.

Our study were some limitations. First, we excluded three rats that died due to their debilitating status because they did not survive until their scheduled sacrifice date. However, because these rats may have represented the most severe corrosive injury cases, this could have biased the results. Nevertheless, we believe these deaths were consequences of severe injury, rather than death related to stricture formation, because the timing of deaths were concentrated in the first week after the corrosive burn. Second, it is possible that peritoneal adhesion formation after surgery may have affected the fluoroscopic results. We used a modified version of Gehanno and Guedon's experimental model, the most commonly used model in previous studies [26]. In this model, the lower esophagus is dissected and ligated to prevent leakage of the caustic substance. Adhesion between the serosa of the esophagus and peritoneum occurred during the healing period. To minimize this adhesion, we meticulously dissected the overlying peritoneum to avoid adjacent tissue damage. Finally, we did not observe interobserver variability regarding the analyses of fluoroscopic and histologic findings.

In conclusion, EW-7197 may be a good alternative treatment option for the prevention of esophageal stricture formation after corrosive burn. Although our results are prospecting, subsequent research is needed to investigate the safety of this TGF- $\beta 1$ inhibitor and to establish an optimized treatment protocol in preventing esophageal strictures after corrosive burns. 


\section{References}

1. Kim, J.H., et al., Corrosive esophageal strictures: long-term effectiveness of balloon dilation in 117 patients. J Vasc Interv Radiol, 2008. 19(5): p. 736-41.

2. Song, H.Y., et al., Corrosive esophageal stricture: safety and effectiveness of balloon dilation. Radiology, 1992. 184(2): p. $373-8$.

3. Arbell, D., et al., Prevention of esophageal strictures in a caustic burn model using halofuginone, an inhibitor of collagen type I synthesis. Laryngoscope, 2005. 115(9): p. 1632-5.

4. Turkyilmaz, Z., et al., Mitomycin C prevents strictures in caustic esophageal burns in rats. J Surg Res, 2005. 123(2): p. $182-7$.

5. Yukselen, V., et al., Colchicine in experimental alkaline burns of the rat esophagus: an old drug, a new indication? Pediatr Surg Int, 2006. 22(4): p. 363-8.

6. Makay, O., et al., Role of allopurinol on oxidative stress in caustic burn: cure for stricture? Pediatr Surg Int, 2007. 23(11): p. 1105-12.

7. Larios-Arceo, F., et al., Protective effects of melatonin against caustic esophageal burn injury in rats. J Pineal Res, 2008. 45(2): p. 21923.

8. Herek, O., et al., Protective effects of ibuprofen against caustic esophageal burn injury in rats. Pediatr Surg Int, 2010. 26(7): p. 721-7.

9. Orozco-Perez, J., et al., Pirfenidone prevents rat esophageal stricture formation. J Surg Res, 2015. 194(2): p. 558-64.

10. Grygielko, E.T., et al., Inhibition of gene markers of fibrosis with a novel inhibitor of transforming growth factor-beta type I receptor kinase in puromycin-induced nephritis. J Pharmacol Exp Ther, 2005. 313(3): p. 943-51.

11. Bonniaud, P., et al., Progressive transforming growth factor beta1-induced lung fibrosis is blocked by an orally active ALK5 kinase inhibitor. Am J Respir Crit Care Med, 2005. 171(8): p. 889-98.

12. de Gouville, A.C., et al., Inhibition of TGF-beta signaling by an ALK5 inhibitor protects rats from dimethylnitrosamine-induced liver fibrosis. Br J Pharmacol, 2005. 145(2): p. 166-77.

13. Moon, J.A., et al., IN-1130, a novel transforming growth factor-beta type I receptor kinase (ALK5) inhibitor, suppresses renal fibrosis in obstructive nephropathy. Kidney Int, 2006. 70(7): p. 1234-43.

14. Kim, J.H., et al., IN-1233, an ALK-5 inhibitor: prevention of granulation tissue formation after bare metallic stent placement in a rat urethral model. Radiology, 2010. 255(1): p. 75-82.

15. Jin, C.H., et al., Discovery of $\mathrm{N}$-((4-([1,2,4]triazolo[1,5-a]pyridin-6-yl)-5-(6-methylpyridin-2-yl)-1H-imidazol-2 -yl)methyl)-2fluoroaniline (EW-7197): a highly potent, selective, and orally bioavailable inhibitor of TGF-beta type I receptor kinase as cancer immunotherapeuticlantifibrotic agent. J Med Chem, 2014. 57(10): p. 4213-38.

16. Krishnaiah, M., et al., Synthesis and biological evaluation of 5-(fluoro-substituted-6-methylpyridin-2-yl)-4-([1,2,4]triazolo[1,5-a]pyridin6-yl )imidazoles as inhibitors of transforming growth factor-beta type I receptor kinase. Bioorg Med Chem Lett, 2015. 25(22): p. 5228-31.

17. Son, J.Y., et al., EW-7197, a novel ALK-5 kinase inhibitor, potently inhibits breast to lung metastasis. Mol Cancer Ther, 2014. 13(7): p. 1704-16.

18. Park, S.A., et al., EW-7197 inhibits hepatic, renal, and pulmonary fibrosis by blocking TGF-beta/Smad and ROS signaling. Cell Mol Life Sci, 2015. 72(10): p. 2023-39.

19. Wijburg, F.A., et al., Nasogastric intubation as sole treatment of caustic esophageal lesions. Ann Otol Rhinol Laryngol, 1985. 94(4 Pt 1): p. 337-41.

20. Zhou, J.H., et al., Management of corrosive esophageal burns in 149 cases. J Thorac Cardiovasc Surg, 2005. 130(2): p. $449-55$.

21. Gumaste, V.V. and P.B. Dave, Ingestion of corrosive substances by adults. Am J Gastroenterol, 1992. 87(1): p. 1-5.

22. Heo, Y.-C., D.-K. Han, and M.T. Kim, Therapeutic effect of local photothermal heating of gold nanoparticle-coated self-expandable metallic stents for suppressing granulation tissue formation in the mouse colon. PLOS ONE, 2021. 16(4): p. e0249530.

23. Jun, E.J., et al., EW-7197, an activin-like kinase 5 inhibitor, suppresses granulation tissue after stent placement in rat esophagus. Gastrointest Endosc, 2017. 86(1): p. 219-228.

24. Border, W.A., et al., Suppression of experimental glomerulonephritis by antiserum against transforming growth factor beta 1. Nature, 1990. 346(6282): p. 371-4.

25. Yeming, W., et al., Balloon catheter dilatation in children with congenital and acquired esophageal anomalies. J Pediatr Surg, 2002. 37(3): p. 398-402.

26. Gehanno, P. and C. Guedon, Inhibition of experimental esophageal lye strictures by penicillamine. Arch Otolaryngol, 1981. 107(3): $\mathrm{p}$. $145-7$. 